\title{
基 \\ Refrigeração de sêmen canino com diluente a base de leite e gema de ovo $2 \%$
}

[Cooling of canine semen with milk-based extender and egg yolk 2\%]

"Artigo Científico/Scientific Article"

\author{
Luisa Lima Nantes Oliveira1 ${ }^{\mathbf{1}}$, Queren Hapuque de Sousa Lima Aguiar ${ }^{\mathbf{1}}$, Fernando Silva Lima ${ }^{\mathbf{1}}$, \\ Larissa Tome Nogueira ${ }^{1}$, Fernanda Carlini Cunha Santos ${ }^{2 *}$
}

\begin{abstract}
${ }^{1}$ Discentes do Curso de Medicina Veterinária, Universidade Federal de Roraima (UFRR), Boa Vista-RR, Brasil.
${ }^{2}$ Líder do Grupo de Pesquisa do Diretório de Grupos de Pesquisa do CNPq: Repro - Reprodução Animal. Departamento de Medicina Veterinária, Universidade Federal de Roraima (UFRR), Boa Vista-RR, Brasil.

*Autora para correspondência/Corresponding author: E-mail: carlini.fernanda@ hotmail.com
\end{abstract}

\section{Resumo}

O processo de conservação de sêmen sob resfriamento necessita de adição de diluentes, contendo diferentes formulações. Estudos com adição de gema de ovo ao diluente apresentam resultados divergentes quanto a qualidade espermática, durante a conservação e criopreservação em diferentes espécies de mamíferos. Diante disso, o objetivo deste experimento foi avaliar a qualidade espermática de sêmen canino adicionado de diluente a base de leite e gema de ovo de galinha a $2 \%$, mantido sob resfriamento a $5^{\circ} \mathrm{C}$ por $72 \mathrm{~h}$. Para isso, 20 ejaculados foram coletados de caninos e submetidos a dois tratamentos: 1) Diluente Sem Gema e 2) Diluente Com Gema de ovo de galinha a $2 \%$. As amostras foram mantidas em geladeira a $5^{\circ} \mathrm{C}$ e avaliadas as 24,48 e $72 \mathrm{~h}$ de resfriamento quanto a motilidade, vigor, integridade de membrana plasmática e morfologia. A análise estatística incluiu estatística descritiva, Shapiro-Wilk, Wilcoxon, análise de variância (ANOVA), comparação de médias pelo teste de Tukey. Os valores foram expressos em a média \pm erro padrão da média. Foi verificada diferença significativa entre os tratamentos quanto à motilidade às $48 \mathrm{~h}$ (Sem Gema 38,3 $\pm 4,2 \%$

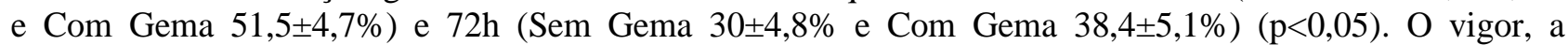
integridade funcional da membrana plasmática e a morfologia espermática não foram significativamente afetados pelo tratamento. Em conclusão, os ejaculados de caninos adicionados de diluente acrescido de gema de ovo de galinha a $2 \%$ apresentaram maior percentual de motilidade durante resfriamento a $5^{\circ} \mathrm{C}$ por $72 \mathrm{~h}$.

Palavras-chave: crioprotetora; ejaculado; espermático; lipoproteína de baixa densidade; resfriamento.

\begin{abstract}
Cooled semen storage requires the addition of extenders containing different formulations. Studies with the addition of egg yolk to the extender presents divergent results regarding sperm quality during cooled and frozen storage in different mammalian species. Therefore, this experiment aimed to evaluate canine sperm quality during cooled storage at $5^{\circ} \mathrm{C}$ for $72 \mathrm{~h}$ in skim-milk extender with $2 \%$ of egg yolk. For that, 20 canine ejaculates were collected and submitted to two treatments: 1) Only Extender, without egg yolk and 2) Extender with chicken Egg yolk 2\%. Samples were kept in a refrigerator at $5^{\circ} \mathrm{C}$ and evaluated at 24; 48 and $72 \mathrm{~h}$ of cooled storage regarding motility, vigor, plasma membrane integrity and sperm morphology. Statistical analysis included descriptive statistics, Shapiro-Wilk, Wilcoxon, analysis of variance (ANOVA), comparison of means by the Tukey test ( $\mathrm{p}<.05)$. Values were expressed by mean \pm standard error mean. About motility, significance difference was observed between treatments at $48 \mathrm{~h}$ (Extender $38.3 \pm 4.2 \%$ e Egg yolk $51.5 \pm 4.7 \%$ ) and $72 \mathrm{~h}$ (Extender $30 \pm 4.8 \%$ e Egg yolk $38.4 \pm 5.1 \%$ ). Vigor, plasma membrane integrity and sperm morphology were unaffected by treatment. In conclusion, canine sperm added with extender $+2 \%$ of chicken egg yolk present higher percentual motility during cooled storage at $5^{\circ} \mathrm{C}$ for $72 \mathrm{~h}$.
\end{abstract}

Keywords: cryoprotectant, low density lipoprotein, refrigeration, sperm, spermatic. 


\section{Introdução}

A redução da temperatura amplia o período de armazenamento de sêmen, aumentando o período de viabilidade espermática, no entanto, este processo de conservação pode resultar em alterações na estrutura e fisiologia celular devido ao choque térmico, danos mecânicos, estresse químico e osmótico (Khalili et al., 2010). Com intuito de reduzir estas ações deletérias, é necessário a adição de diluente de sêmen, sendo que este deve ser atóxico, isotônico, com poder tamponante, atuar na prevenção de crescimento microbiano, minimização de danos mecânicos e como fonte de nutrientes (Senger, 2005).

Múltiplas substâncias podem ser adicionadas ao diluente com intuito de melhorar a eficiência na preservação da qualidade espermática durante resfriamento. Neste contexto, a gema do ovo possui proteínas de alto valor biológico, ácidos graxos essenciais, fosfolipídeos e glicerol de grande aplicação industrial e biomédica. Estudos têm demonstrado que as lipoproteínas de baixa densidade são responsáveis por proteger a célula do choque térmico, auxiliar na preservação da motilidade, da integridade do DNA nuclear e das membranas espermáticas (Manjunath et al., 2002; Moussa et al., 2002). Além disso, a gema de ovo apresenta baixo custo, alta disponibilidade e fácil aquisição.

Estudos com adição de gema de ovo ao diluente apresentam resultados divergentes quando a qualidade espermática durante a conservação e criopreservação em diferentes espécies de mamíferos, incluindo suínos (Bathgate et al., 2006; Toniolli et al., 2016); bovinos (Wall e Foot, 1999); caprinos (Viana et al., 2006; Santiago-Moreno et al., 2008); búfalos (Andrabi et al., 2008), equinos (Bruemmert et al., 2002; Melo e Henry, 2005) e caninos (Silva et al., 2000; Iguer-Ouada e Verstegen, 2001; Moura et al., 2002; Cardoso et al., 2010). Além disso, a maioria dos trabalhos mencionou o uso de $20 \%$ de gema (Silva, 2000; Iguer-Ouada e Verstegen, 2001; Moura et al., 2002; Cardoso et al., 2010; Bispo et al., 2011), independente da composição do diluente base. Evidências laboratoriais indicam que uma alta concentração de gema possa interferir no resultado de avaliações bioquímicas e metabólicas (Wall e Foot, 1999), sendo que uma possível alternativa seria reduzir a quantidade, exercendo ação benéfica sobre os parâmetros espermáticos. Em outras espécies, como equinos, a adição de gema de ovo em baixa concentração
(2\%) durante processo de criopreservação já é uma realidade (Pillet et al., 2008). Em caninos, Gloria et al. (2020) reportaram que o uso de gema de ovo na concentração de 5 e 10\% durante criopreservação de sêmen canino resultou em maiores médias de viabilidade espermática após descongelamento, quando comparados ao diluente contendo $20 \%$ de gema.

Neste contexto, o objetivo do presente trabalho foi avaliar a qualidade espermática de sêmen canino adicionado de diluente a base de leite e gema de ovo de galinha a $2 \%$, mantido sob resfriamento a $5^{\circ} \mathrm{C}$ por $72 \mathrm{~h}$.

\section{Materiais e Métodos}

O experimento foi conduzido na Universidade Federal de Roraima (UFRR), Boa Vista, Roraima. Foram coletados ejaculados de 20 cães, de diferentes raças (Shit $\mathrm{Zu}$, Golden Retriver, Pug, Pit Bull, Daschund, Beagle, Buldogue), adultos (acima de 1 ano), com bom estado corporal e hígidos, sendo realizada uma coleta por animal $(n=20)$.

Para obtenção dos ejaculados, foi utilizado o método de mão enluvada. Os ejaculados foram avaliados quanto a parâmetros macroscópicos (cor, volume, odor e aspecto) e microscópicos (concentração espermática, motilidade, vigor, teste hiposmótico e morfologia espermática), seguindo a metodologia do CBRA (2013).

Após avaliação dos parâmetros, foi adicionado diluente Kenney (4,9 g de glicose, $2,4 \mathrm{~g}$ leite em pó desnatado em $100 \mathrm{~mL}$ água destilada, $\mathrm{pH} 6,7$ e osmolaridade $330 \mathrm{mOsm} / \mathrm{kg}$ ) resultando em concentração final de $25 \times 10^{6}$ espermatozoides $/ \mathrm{mL}$, de acordo com os tratamentos: 1) Diluente Sem Gema e 2) Diluente Com Gema de ovo de galinha a $2 \%$.

As amostras foram mantidas resfriadas em geladeira a $5^{\circ} \mathrm{C}$ por $72 \mathrm{~h}$ e avaliadas a cada $24 \mathrm{~h}$. Para avaliação, uma alíquota de cada tratamento foi mantida em banho-maria a $37^{\circ} \mathrm{C}$ por 5 minutos para as análises microscópicas, incluindo motilidade, vigor, morfologia espermática e teste hiposmótico (Figura 1).

A motilidade foi avaliada indicando percentual de espermatozoides móveis, em escala de $0-100$. O vigor indicando força de movimento das células, em escala de 0 (ausência de movimento) e 5 (movimento vigoroso). A concentração espermática foi avaliada através da contagem em câmara de Neubauer. A avaliação da morfologia espermática foi realizada por 
coloração com eosina, onde 100 células espermáticas foram contadas e classificadas quanto a defeitos maiores (cabeça anormal, gota citoplasmática proximal e cauda fortemente enrolada) e defeitos menores (cabeça isolada anormal, gota citoplasmática distal e cauda dobrada). O teste hiposmótico foi realizado pela adição de $10 \mu \mathrm{L}$ de sêmen e $20 \mu \mathrm{L}$ de água destilada, incubados por $30 \mathrm{~min}$ a $37^{\circ} \mathrm{C}$ e contagem de 100 células, classificadas em cauda enrolada (membrana funcional) e cauda reta (membrana afuncional). As análises foram realizadas com auxílio de microscópio ótico (BX 41 Olympus América, Inc, São Paulo, SP, Brasil), sempre pela mesma pessoa previamente treinada.

A análise estatística foi realizada com auxílio do programa de computador Statistic $9^{\circledR}$ (Analytical Software, Tallahassee, FL, USA), com nível de significância $<0,05$, incluindo estatística descritiva, teste de normalidade Shapiro-Wilk, Wilcoxon, análise de variância (ANOVA) e comparação de médias pelo teste de Tukey.

\section{Resultados e Discussão}

Imediatamente após coleta, todos os ejaculados apresentaram valores de qualidade espermática acima dos mínimos preconizados pelo CBRA (2013). No sêmen fresco, a média \pm erro padrão da média de motilidade foi de $79,5 \pm 2,6 \%$; vigor $3 \pm 0,1$; hiposmótico $81,5 \pm 2,8 \%$; morfologia normal $85,4 \pm 1,3 \%$; defeitos totais $14 \pm 1,4 \%$; defeitos maiores $8,5 \pm 1,1 \%$ e defeitos menores $6,3 \pm 0,8 \%$.

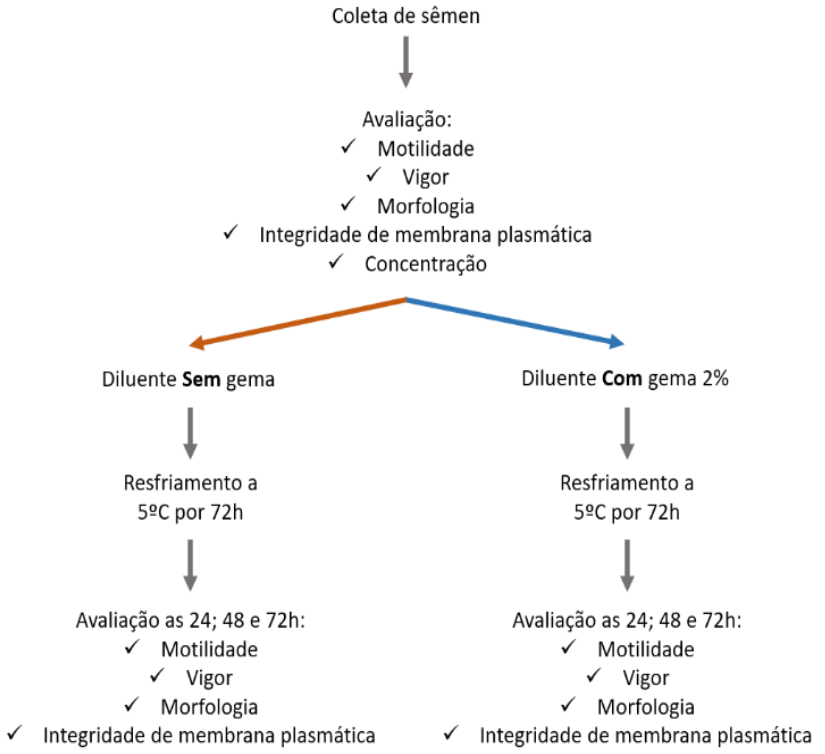

Figura 1. Representação esquemática da manipulação de sêmen canino submetido aos tratamentos com diluente sem gema ou com gema de ovo a $2 \%$.

Durante o período de resfriamento, foi observado redução na motilidade, vigor, hiposmótico e morfologia em ambos tratamentos. Foi verificada diferença significativa entre os tratamentos quanto à motilidade às $48 \mathrm{~h}$ (Sem Gema $38,3 \pm 4,2 \%$ e Com Gema $51,5 \pm 4,7 \%$ ) e $72 \mathrm{~h}$ (Sem Gema $30 \pm 4,8 \%$ e Com Gema $38,4 \pm 5,1 \%$ ) $(\mathrm{p}<0,05)$. O tratamento Com Gema apresentou maiores valores médios de vigor, hiposmótico e morfologia às $24 \mathrm{~h}, 48 \mathrm{~h}$ e $72 \mathrm{~h}$, em comparação ao Sem Gema ( $>0,05)$ (Tabela 1).

Tabela 1. Parâmetros microscópicos (média \pm erro padrão da média) de sêmen canino ( $\mathrm{n}=20$ ), conforme tratamento com diluente sem gema de ovo (Sem) e com gema de ovo de galinha a $2 \%$ (Gema), durante resfriamento a $5^{\circ} \mathrm{C}$ por $72 \mathrm{~h}$.

\begin{tabular}{ccccccc}
\hline Parâmetros & $\begin{array}{c}\text { Sem } \\
\mathbf{2 4 h}\end{array}$ & $\begin{array}{c}\text { Gema } \\
\mathbf{2 4 h}\end{array}$ & $\begin{array}{c}\text { Sem } \\
\mathbf{4 8 h}\end{array}$ & $\begin{array}{c}\text { Gema } \\
\mathbf{4 8 h}\end{array}$ & $\begin{array}{c}\text { Sem } \\
\mathbf{7 2 h}\end{array}$ & $\begin{array}{c}\text { Gema } \\
\mathbf{7 2 h}\end{array}$ \\
\hline Motilidade & $53,4 \pm 4,5$ & $61 \pm 5,0$ & $38,3 \pm 4,2^{\text {a }}$ & $51,5 \pm 4,7^{\mathrm{b}}$ & $30 \pm 4,8^{\mathrm{a}}$ & $38,4 \pm 5,1^{\mathrm{b}}$ \\
Vigor & $2,6 \pm 0,1$ & $2,8 \pm 0,1$ & $2 \pm 0,1$ & $2,4 \pm 0,1$ & $1,6 \pm 0,2$ & $2 \pm 0,2$ \\
Hiposmótico & $75,5 \pm 2,5$ & $78 \pm 2,5$ & $73,3 \pm 2,4$ & $74,2 \pm 2,3$ & $70,7 \pm 2$ & $74,1 \pm 2$ \\
Morfologia & $79 \pm 1,6$ & $80,7 \pm 1,4$ & $74 \pm 1,4$ & $77,1 \pm 1,6$ & $72 \pm 1,8$ & $76,6 \pm 2$ \\
Defeitos & $21 \pm 1,6$ & $19,2 \pm 1,4$ & $25 \pm 1,2$ & $23,4 \pm 1,7$ & $27,7 \pm 1,8$ & $23,3 \pm 2$ \\
Defeito maior & $13,2 \pm 1,4$ & $12,3 \pm 1,3$ & $14,5 \pm 1$ & $13,1 \pm 1,2$ & $15,8 \pm 1,4$ & $12,8 \pm 1$ \\
Defeito menor & $7,7 \pm 0,8$ & $6,8 \pm 0,9$ & $10,9 \pm 0,9$ & $9,7 \pm 0,7$ & $11,7 \pm 1,2$ & $10,4 \pm 1,2$ \\
\hline
\end{tabular}

a,b indicam diferença significativa entre os tratamentos em um mesmo momento ( $p<0,05$ ).

Concordando com o presente experimento, o efeito benéfico de gema de ovo na preservação de motilidade espermática de caninos foi demonstrado previamente por Iguer-Ouada e
Verstegen (2001). Estes autores também demonstraram que a gema apresenta um efeito protetor sobre o acrossoma, sendo observado melhor conservação da integridade acrossomal e 
prevenção da ocorrência da reação acrossômica espontânea nos diluidores suplementados com $20 \%$ de gema de ovo (Iguer-Ouada e Verstegen, 2001). Moura et al. (2002) também reportaram que a motilidade e vigor dos meios com Tris acrescido de $20 \%$ gema de ovo, tanto de galinha como de codorna, apresentaram significativamente maiores valores após resfriamento, quando comparados ao grupo sem gema.

A principal hipótese para ação protetora da gema é a ocorrência de interação específica entre lipoproteínas de baixa densidade do ovo com determinadas proteínas do líquido seminal (Manjunath et al., 2002; Bencharif et al, 2010). Esta interação promoveria a diminuição da perda de colesterol e fosfolipídeos da membrana dos espermatozoides, prevenindo a capacitação prematura e subsequente reação do acrossoma (Bergeron et al., 2004). Foulkes (1977), estudando a interação de componentes da gema com espermatozoides bovinos congelados, propôs que tais componentes se ligariam firmemente à membrana espermática, exercendo papel protetor ao estabilizá-la durante o resfriamento ou ao conseguir manter a pressão coloidal do meio externo.

Em suínos, Toniolli et al. (2016) compararam diferentes concentrações de gema de ovo em pó $(1 ; 3 ; 5$ e $7 \%)$, adicionada ao diluente a base de água de coco, e relataram que os tratamentos contendo gema apresentaram maiores valores de motilidade após $24 \mathrm{~h}$ de resfriamento a $17^{\circ} \mathrm{C}$.

Em caprinos, Bispo et al. (2011) compararam duas concentrações de gema de ovo $(2,5 \%$ e $20 \%)$ adicionado ao diluente citrato-gema e relataram que para as características in vitro de motilidade, vigor, integridade e funcionalidade de membrana, o diluente com baixa concentração $(2,5 \%)$ apresentou maiores valores médios após $24 \mathrm{~h}$ de resfriamento a $5^{\circ} \mathrm{C}$. Viana et al. (2006) constataram que o diluente tris-gema apresentou melhor qualidade espermática, referente a motilidade total, vigor e patologias de acrossoma, quando comparado ao diluente leite desnatadoglicose (sem gema) durante resfriamento de sêmen caprino a $4^{\circ} \mathrm{C}$. No presente trabalho, o tratamento com adição de gema em baixa concentração também apresentou melhor qualidade espermática durante resfriamento, quando comparado ao sem gema.
A adição de 3 e $10 \%$ de gema ao diluente preservaram melhor a membrana plasmática de espermatozoides em equinos durante resfriamento, quando comparado ao diluente sem gema. O meio contendo 3\% de gema apresentou maior motilidade, seguido pelo $10 \%$ e, por último, o sem gema (Melo et al., 2005).

Em discordância com nossos dados, Cardoso et al. (2010) relataram que a inclusão de gema de ovo (5; 10 e $20 \%$ ), mesmo na menor concentração, foi suficiente para promover a acidificação do meio diluidor ( $\mathrm{pH}$ inferior a 7), o que não ocorreu no diluente controle (sem gema), durante resfriamento de sêmen canino por 5 dias a $4^{\circ} \mathrm{C}$. Este efeito não foi verificado no presente estudo, em que o pH do diluente base foi de 6,7, no início do experimento. Considerando que a gema de ovo é um produto de origem animal e rapidamente perecível, uma possível explicação para o pior desempenho de diluentes com altas concentrações deste produto poderia ser devido ao estado de deterioração.

O efeito benéfico ou prejudicial da adição de gema de ovo ao diluente de sêmen canino é bastante divergente na literatura, podendo estar associados a diferentes fatores, incluindo tipo de diluente, concentração de gema de ovo, curva de resfriamento, características individuais dos cães, entre outros. Estudos sugerem que a concentração ótima de proteínas de baixa densidade, presentes na gema de ovo, para preservação de sêmen seja espécie específica (Gloria et al., 2020). Além disso, a gema de ovo é um produto biológico com composição complexa, sendo que esta composição pode diferir entre lotes, por múltiplos fatores, dificultando ainda mais a padronização de diluidores contendo gema (Aurich, 2005).

A quantidade adequada de gema a ser adicionada ao diluente também não está completamente esclarecida. Embora a maioria dos autores utilize $20 \%$, os resultados do presente experimento sugerem que a adição de $2 \%$ de gema afetou positivamente a motilidade de sêmen canino durante resfriamento a $5^{\circ} \mathrm{C}$ por $72 \mathrm{~h}$ e não influenciou a integridade funcional da membrana plasmática nem quantidade de defeitos morfológicos.

\section{Conclusão}

Os ejaculados adicionados de diluente acrescido de gema de ovo a $2 \%$ apresentaram maior percentual de motilidade durante resfriamento a $5^{\circ} \mathrm{C}$ por $72 \mathrm{~h}$ em caninos. 


\section{Conflito de Interesse}

Declaramos não existir conflitos de interesse.

\section{Comitê de Ética}

Este experimento utilizou animais como modelo experimental sendo aprovado pelo Comitê de Ética no Uso de Animais (CEUA-UFRR) sob registro 06/2018.

\section{Agradecimentos}

Agradecemos ao CNPq e a Universidade Federal de Roraima, pelo apoio financeiro, e ao Repro - Grupo de Ensino, Pesquisa e Extensão em Reprodução Animal, pelo trabalho em equipe.

\section{Referências}

Andrabi, S.M.H.; Ansari, M.S.; Ullah, N.; Anwar, M.; Mehmood, A.; Akhter, S. Duck egg yolk in extender improves the freezeability of buffalo bull spermatozoa. Animal Reproduction Science, 104(2-4): 427-433, 2008.

Aurich, C. Factors affecting the plasma membrane function of cooled-stored stallion spermatozoa. Animal Reproduction Science, 89(1-4): 6575, 2005.

Bathgate, R.; Maxwell, W.M.C.; Evans, G. Studies on the effect supplementing boar semen cryopreservation media with different avian egg yolk types on in vitro post-thaw sperm quality. Reproduction in Domestic Animals, 41(1): 68-73, 2006.

Bencharif, D.; Amirat-Briand, L.; Garand, A.; Anton, M.; Schmitt, E.; Desherces, S. Freezing canine sperm: comparison of semen extenders containing Equex and LDL (Low Density Lipoproteins). Animal Reproduction Science, 119(3-4): 305-313, 2010.

Bergeron, A.; Crete, M.H.; Brindle, Y.; Manjunath, P. Low-density lipoprotein fraction from hen's egg yolk decreases the binding of the major proteins of bovine seminal plasma to sperm and prevents lipid efflux from the sperm membrane. Biology of Reproduction, 70(3): 708-717, 2004.

Bispo, C.A.S.; Pugliesi, G.; Palhão, M.P.; Coelho, P.G.B.; Ker, P.G.; Rodrigues, M.T.; Carvalho, G.R. Características in vitro e fertilidade do sêmen caprino armazenado a $5^{\circ} \mathrm{C}$ por 24 horas utilizando duas concentrações de gema de ovo no diluente. Ciência Animal Brasileira, 12(4): 653-660, 2011.
Bruemmert, J.E.; Coy, R.C.; Squires, E.L.; Graham, J.K. Effect of pyruvate on the function of stallion spermatozoa stored for up to 48 hours. Journal of Animal Science, 80(1): 12-18, 2002.

Cardoso, J.F.S.; Paula, N.R.O.; Uchoa, D.C.; Silva, L.D.M. Diferentes concentrações de gema de ovo na qualidade do sêmen canino diluído em ACP®-106 e resfriado a $4^{\circ} \mathrm{C}$. Comunicata Scientiae, 1(2): 146-152, 2010.

Colégio Brasileiro de Reprodução Animal (CBRA). Manual para exame andrológico e avaliação de sêmen animal. $3^{\mathrm{a}}$ ed. Belo Horizonte: CBRA, 2013. 104p.

Foulkes, J.A. The separation of lipoproteins from egg yolk and their effect on the motility and integrity of bovine spermatozoa. Journal of Reproduction and Fertility, 49(2): 277-284, 1977.

Gloria, A.; Zambelli, D.; Carluccioa, A.; Cunto, M.; Ponzio, P.; Contrid, A. Is the protective effect of egg yolk against osmotic and cryogenic damage on dog spermatozoa dosedependent? Animal Reproduction Science, 213(106259):1-11, 2020.

Iguer-Ouada, M.; Verstegen, J.P. Long-term preservation of chilled canine semen: effect of commercial and laboratory prepared extenders. Theriogenology, 55(2): 671-684, 2001.

Khalili, B.; Jafaroghli, M.; Farshad, A.; PareshKhiavi, M. The effects of different concentrations of glycine and cysteine on the freezability of Moghani ram spermatozoa. Asian-Australasian Journal of Animal Sciences, 23(3): 318- 325, 2010.

Manjunath, P.; Nauc, V.; Bergeron, A.; Menard, M. Major proteins of bovine seminal plasma bind to the low-density lipoprotein fraction of hen's egg yolk. Biology of Reproduction, 67(4): 1250-1258, 2002.

Melo, M.I.V.; Henry, M.; Beker, A.R.C. Teste hiposmótico na avaliação do sêmen eqüino resfriado com diferentes diluidores. Arquivo Brasileiro de Medicina Veterinária e Zootecnia, 57(6): 757-763, 2005.

Moura, C.S.; Cavalcanti, M.C.O.; Guerra, M.M.P.; Batista, A.M.; Barreto, M.B.P. Teste de avaliação in vitro e criopreservação do sêmen do cão utilizando diferentes diluidores. Revista Brasileira de Ciência Veterinária, 9(2): 102-106, 2002.

Moussa, M.; Martinet, V.; Trimeche, A.; Tainturier, D.; Anton, M. Low density 
lipoproteins extracted from hen egg yolk by an easy method: cryoprotective effect on frozen thawed bull semen. Theriogenology, 57(6): 1695-1706, 2002.

Pillet, E.; Batellier, F.; Duchamp, G.; Furstoss, V.; Le Vern, Y.; Kerboeuf, D.; Vidament, M.; Magistrini, M. Freezing stallion semen in INRA96®-based extender improves fertility rates in comparison with INRA82. Dairy Science \& Technology, 88(2): 257-265, 2008.

Santiago-Moreno, J.; Coloma, M.A.; ToledanoDíaz, A.; Gómez-Brunet, A.; Pulido-Pastor, A.; Zamora-Soria, A.; Carrizosa, J.A.; Urrutia, B.; López-Sebastián, A. A comparison of the protective action of chicken and quail egg yolk in the cryopreservation of Spanish ibex epididymal spermatozoa. Cryobiology, 57(1): 25-29, 2008.

Senger, P.L. Pathways to pregnancy \& parturition. $3^{\text {rd }}$ ed. Redmon: Current Conceptions. Inc, 2012. 381p.

Silva, A.R.; Cardoso, R.C.S.; Silva, L.D.M.
Congelação de sêmen canino com diferentes concentrações de gema de ovo e glicerol em diluidores à base de tris e água de coco. Ciência Rural, 30(6):1021-1025, 2000.

Toniolli, R.; Barros, T.B.; Toniolli, L.S.; Guimarães, D.B.; Freitas, E.N.; Nunes, T.G.P. Diferentes concentrações de gema de ovo em pó adicionada ao diluente acp-103® na conservação do sêmen suíno. Ciência Animal Brasileira, 17(2): 243-251, 2016.

Viana, A.K.S.; Chalhoub, M.; Ribeiro Filho, A.L.; Almeida, A.K.; Portela, A.P.M.; Bittencourt, R.F.; Alves, S.G.G.; Bittencourt, T.C.C.; Quintela, A.T. Avaliação in vitro do sêmen caprino resfriado, com ou sem centrifugação do plasma seminal e diluído em leite desnatado-glicose e tris-gema de ovo. Ciência Animal Brasileira, 7(1): 67-76, 2006.

Wall, R.J.; Foote, R.H. Fertility of bull semen frozen and store in clarified egg yolk-Trisglycerol extender. Journal of Dairy Science, 82(4): 817-821, 1999. 